\title{
Characterization of changes in total body composition for patients with head and neck cancer undergoing chemoradiotherapy using dual-energy X-ray absorptiometry
}

\author{
William Jackson, BS, ${ }^{1}$ Neil Alexander, MD, ${ }^{2,3,4,5,8}$ Matthew Schipper, PhD, ${ }^{1}$ Lorraine Fig, MD, ${ }^{6}$ Felix Feng, MD, ${ }^{1}$ Shruti Jolly, $\mathrm{MD}^{1,7^{*}}$ \\ ${ }^{1}$ Department of Radiation Oncology, University of Michigan, Ann Arbor, Michigan, ${ }^{2}$ Department of Internal Medicine, University of Michigan, Ann Arbor, Michigan, ${ }^{3}$ Division of \\ Geriatric and Palliative Medicine, University of Michigan, Ann Arbor, Michigan, ${ }^{4}$ Mobility Research Center, University of Michigan, Ann Arbor, Michigan, ${ }^{5}$ nstitute of Gerontology, \\ University of Michigan, Ann Arbor, Michigan, ${ }^{6}$ Department of Nuclear Medicine, VA Medical Center, Ann Arbor, Michigan, ${ }^{7}$ Department of Radiation Oncology, VA Medical Center, \\ Ann Arbor, Michigan, ${ }^{8}$ Geriatric Research, Education, and Clinical Center (GRECC), VA Medical Center, Ann Arbor, Michigan.
}

Published online 21 November 2013 in Wiley Online Library (wileyonlinelibrary.com). D0I 10.1002/hed.23461

\begin{abstract}
Background. Patients with head and neck cancer experience significant weight loss secondary to concurrent chemoradiotherapy (CCRT). Using dual-energy X-ray absorptiometry (DEXA) scans, we characterize total body composition changes during and after CCRT in order to develop novel clinical care models that will improve the patient's quality of life (QOL).

Methods. Sixty DEXA scans were obtained from 12 patients undergoing CCRT for locally advanced squamous cell head and neck cancer. DEXAs were performed at baseline, during treatment, completion of CCRT, and then 1 and 2 months posttreatment.

Results. Mean weight loss by treatment end was $9.5 \mathrm{~kg}(10.2 \%$; $p=.0002$ ). On average, lean body mass (LBM) decreased $10.2 \%$ $(p=.001)$, and fat body mass (FBM) decreased $11.1 \%(p=.001)$ during
\end{abstract}

CCRT. LBM began to normalize after completion of treatment, whereas FBM continued to decline.

Conclusion. Substantial loss of muscle and FBM occurs in patients undergoing CCRT for head and neck cancer. To prevent long-term disability and QOL decline after curative CCRT, clinical care interventions incorporating aggressive nutrition/exercise counseling are needed. (c) 2013 Wiley Periodicals, Inc. Head Neck 36: 1356-1362, 2014

KEY WORDS: total body composition, lean body mass, dual-energy $x$-ray absorptiometry (DEXA), head and neck cancer, chemoradiotherapy

\section{INTRODUCTION}

Changes in total body composition are common in patients with cancer over the course of their treatment. Cancers associated with adverse changes in body composition include pancreatic cancer, gastrointestinal cancers, esophagogastric cancer, ovarian cancer, non-small cell lung cancer, prostate cancer, breast cancer, and head and neck cancer. ${ }^{1-9}$ Specific changes in total body composition tend to vary according to disease site and corresponding treatment modality. Patients treated for breast and prostate cancer can undergo sarcopenic obesity, which results from a loss of muscle mass with an increase in FBM. ${ }^{8,9}$ Patients with advanced cancers often experi-

*Corresponding author: S. Jolly, The University of Michigan Medical Center, 1500 East Medical Center Drive, Ann Arbor, Ml 48105. E-mail: shrutij@med.umich.edu

Contract grant sponsor: Supported in part by G. Clare Bishop M.D. Memorial, Jerome and Mary Miller Cancer, and Endowment for Discovery Funds at the University of Michigan Comprehensive Cancer Center, VA Rehabilitation Research and Development, and the Office of Research and Development of the Department of Veterans Affairs. The authors also acknowledge support of the National Institute of Health Claude Pepper Center (AG024824), and the Dorothy and Herman Miller Fund for Mobility Research in Older Adults. Dr. Alexander is a recipient of the K24 Mid-Career Investigator Award in PatientOriented Research AG109675 from the National Institute on Aging. ence cancer cachexia, which presents as a drastic loss in muscle mass, with or without a corresponding loss of FBM. ${ }^{10}$ Importantly, these changes in body composition are known to be associated with decreased quality of life (QOL) and worse overall prognosis. ${ }^{10-14}$

Patients with head and neck cancers often experience significant unintentional weight loss that is multifactorial in nature. ${ }^{15}$ It has been suggested that approximately $70 \%$ of this weight loss arises from a loss of lean body mass $(\mathrm{LBM}),{ }^{6}$ and that this loss occurs despite appropriate nutritional intake. ${ }^{16}$ Furthermore, this weight loss has been shown to predict decreased overall survival in patients with head and neck cancer. ${ }^{17,18}$ Therefore, in order to improve QOL, physical functional status, and potentially to improve overall prognosis, understanding the changes that occur during and after head and neck cancer treatment are essential to developing clinical models to help avert weight loss and changes in total body composition in these patients.

To date, relatively little is known regarding the changes in total body composition that occur during and after treatment of head and neck cancer. We sought to characterize changes in total body composition for patients undergoing concurrent chemoradiation therapy (CCRT) for locally advanced squamous cell head and neck cancer using serial total body dual-energy X-ray absorptiometry 
(DEXA) scans. Because patients with head and neck cancer treated with CCRT often experience significant dehydration, or changes in total fluid volume, we also sought to correlate changes in total body composition with hydration status through analysis of serum creatinine levels.

\section{MATERIALS AND METHODS}

\section{Patient selection}

Through an institutional review board approved prospective study assessing the impact of exercise versus usual care on changes in total body composition, assessed using DEXA, for patients receiving CCRT for locally advanced squamous cell head and neck cancer, 12 patients were accrued after giving informed consent. Eligibility criteria included patients with American Joint Committee on Cancer stage II to IV squamous cell head and neck cancer receiving CCRT as first-line treatment without surgery. Patients were not eligible if they had active cardiopulmonary disease, dementia, acute medical conditions unrelated to their head and neck cancer, refused a percutaneous endoscopic gastrostomy tube for nutrition as determined necessary by the treating physician, or were actively receiving physical therapy or exercising 2 or more hours per day. All patients underwent a preliminary nutritional assessment by a dietician before initiation of treatment. Six patients received a percutaneous endoscopic gastrostomy (PEG) tube before initiation of radiation therapy, and an additional 3 patients received a PEG tube during radiation therapy for enteral nutrition. Patients were monitored throughout their treatment to ensure appropriate nutritional status with weekly assessments during treatment in the radiation oncology clinic. If a patient experienced $>5 \%$ to $10 \%$ decrease in body mass during the course of treatment, a nutritionist was consulted. Eight patients met with a nutritionist at least once after the initiation of treatment. Finally, because no statistically significant differences in total body composition were noted between the exercise and usual care groups, we have combined their total body composition analysis for the purposes of this study to better understand these changes. All procedures were in accordance with the ethical standards of the committee on human experimentation of the institution or in accord with the Helsinki Declaration of 1975 as revised in 1983.

\section{Treatment and follow-up}

Patients were treated with platinum-based CCRT for a total of 7 weeks to a total dose of 70 Gy using intensitymodulated radiation therapy (IMRT). Treatment was administered to the primary tumor and bilateral necks. Patients received IMRT at 2 institutions, with 9 patients receiving treatment at the VA Ann Arbor Healthcare System, and 3 receiving treatment at the University of Michigan. CCRT lasted 7 weeks. Patients were seen in followup at 1 and 2 months post-CCRT. Serum creatinine was routinely measured during and after CCRT to assess kidney function before platinum-based chemotherapy and serve as a means to assess fluid status.

\section{Dual-energy X-ray absorptiometry}

Total body DEXA scans were performed using an iDXA whole body scanner (GE/Lunar Corp, Madison, WI). All DEXA scans were performed at 1 institution using the same scanner. DEXA scans were performed with the patients in the supine position on the imaging table and were acquired and processed in accord with the manufacturer's protocol. All patients received a DEXA scan before the initiation of CCRT (week 0), during treatment (between weeks 3 and 5), at the completion of treatment (week 7), 4 weeks posttreatment (1-month followup), and again at 7 to 8 weeks posttreatment (2-month follow-up).

\section{Statistical analysis}

The cohort for the present analysis is a subset of a larger cohort that is being accrued for a study assessing the impact of exercise training on changes in total body composition for patients receiving CCRT for locally advanced head and neck cancer. Because the present analysis was only being performed on a small subset of the cohort from the exercise training study, our goal was not to assess the impact of exercise training, but rather to characterize the overall changes in total body composition for the 12 patients who had completed CCRT for locally advanced head and neck cancer to date. These patients had been randomized to receive either exercise training or usual care. However, as no significant differences were observed between patients assigned to exercise $(n=7)$ versus those assigned to usual care $(n=5)$ regarding mean decrease in LBM from baseline to the end of CCRT $(7.1 \mathrm{~kg}$ vs $4.6 \mathrm{~kg} ; p=.4)$ or mean decrease in FBM over this same time period $(3.0 \mathrm{~kg}$ vs $4.0 \mathrm{~kg}$; $p=.5)$, they were analyzed together. Mean cohort DEXA values from different points in time were compared using the paired samples $t$ test. Pearson correlation coefficients $(r)$ were calculated to quantify correlation between variables of interest. All statistical analysis was performed using MedCalc (v12.3.0.0, MedCalc Software, Mariakerke, Belgium).

\section{RESULTS}

\section{Patient characteristics and changes in total body mass}

All 12 patients had completed treatment at the time of analysis. Eleven of 12 patients had been seen for their 2month posttreatment follow-up. Baseline and treatmentrelated characteristics for all patients can be found in Table 1. Median age of the cohort was 59 years at the initiation of CCRT. All 12 patients were men, as the majority was accrued from the Veteran's Affairs (VA) hospital. Ten of 12 patients had tumors arising in the oropharynx, with the most common site being the base of the tongue, and the majority of tumors were stage IVA (75\%). Changes in total body mass (TBM), LBM, and FBM from baseline through treatment, to the 2-month posttreatment follow-up can be found in Figure 1. Mean pretreatment (baseline) weight was $93.0 \mathrm{~kg}$ (standard deviation $\pm 12.6 \mathrm{~kg} ; 99.0 \pm 10.8$ control vs $88.7 \pm 12.7$ exercise), and mean pretreatment body mass index (BMI) was $29.6( \pm 3.7 ; \quad 28.3 \pm 2.4$ control vs $26.2 \pm 3.2$ 
TABLE 1. Patient baseline and treatment related characteristics.

\begin{tabular}{lcc}
\hline Characteristic & Mean $( \pm$ SD) & No. of patients (\%) \\
\hline Age, y & $57.0( \pm 8.1)$ & \\
Weight, kg & $93.0( \pm 12.6)$ & \\
BMI & $29.6( \pm 3.7)$ & \\
LBM, kg & $59.2( \pm 7.8)$ & \\
FBM, kg & $30.6( \pm 7.5)$ & \\
Stage & & $2(16.7)$ \\
III & & $9(75.0)$ \\
IVA & & $1(8.3)$ \\
IVB & & $6(50.0)$ \\
Tumor site & & $4(33.3)$ \\
Base of tongue & & $18.3)$ \\
Tonsil & & $1(8.3)$ \\
Aryepiglottic fold & & $12(100.0)$ \\
Unknown primary & & \\
Treatment modality & & $12(100.0)$ \\
CCRT & & \\
IMRT dose, Gy & & $9(75.0)$ \\
RT treatment target & & $6(50.0)$ \\
Primary tumor + bilateral neck & & $3(25.0)$ \\
Receiving PEG & & \\
Total & & \\
At baseline & & \\
During treatment & & \\
\hline
\end{tabular}

Abbreviations: BMI, body mass index; LBM, lean body mass; FBM, fat body mass; CCRT, concurrent chemoradiotherapy; IMRT, intensity-modulated radiotherapy; RT, radiotherapy; PEG, percutaneous endoscopic gastrostomy.

exercise). Eleven of 12 patients lost weight over the 7week course of CCRT, 5 from the control arm and 6 from the exercise arm. Mean weight loss by the end of treatment was $9.5 \mathrm{~kg}(10.2 \%$ decrease from baseline; $p=.0002)$, resulting in a mean cohort weight of $83.5 \mathrm{~kg}$ $( \pm 10.1 \mathrm{~kg} ; 90.4 \pm 6.5$ control vs $78.6 \pm 9.5$ exercise) at the end of CCRT, and a mean BMI of $26.5( \pm 2.8$; $28.0 \pm 2.6$ control vs $25.4 \pm 2.6$ exercise). The largest weight loss seen at the end of CCRT was $17.6 \mathrm{~kg}$ (exercise arm). One patient in the exercise arm gained $1.9 \mathrm{~kg}$ from the initiation of treatment to the end of treatment. By the 2-month posttreatment follow-up, half of the patients continued to have declines in their weight, whereas the remaining had either stabilized or begun to regain weight, resulting in a mean cohort weight of 81.1 $\mathrm{kg}(12.8 \%$ decrease from baseline; $p=.0003 ; 87.0 \pm 7.3$

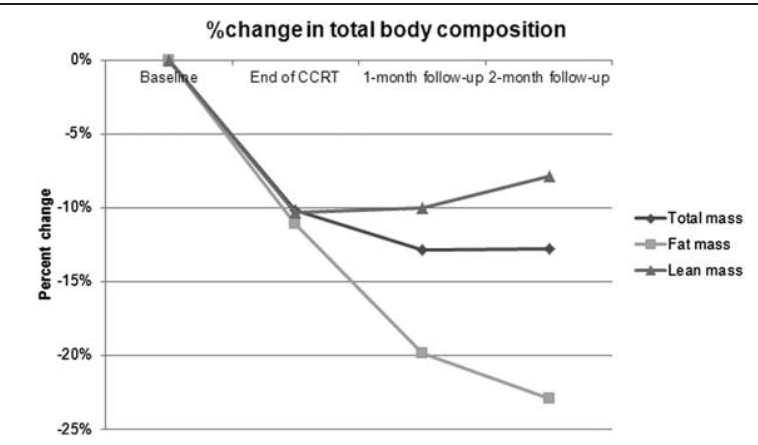

FIGURE 1. Percent change in total, lean, and fat body mass from baseline through 2-month posttreatment follow-up. control vs $76.3 \pm 10.3$ exercise) at the 2 -month posttreatment follow-up, and a mean BMI of 26.0 ( \pm 2.8 ; $27.0 \pm 2.9$ control vs $25.2 \pm 2.9$ exercise).

\section{Changes in lean body mass}

Mean LBM at baseline was $59.2 \mathrm{~kg}( \pm 7.8 \mathrm{~kg}$; $60.9 \pm 8.4$ control vs $57.9 \pm 7.9$ exercise), and accounted for $64 \%$ of mean TBM. On average, patients lost $10.2 \%$ ( $p=.001)$ of their LBM over the course of CCRT, such that the mean LBM at the end of treatment was $53.1 \mathrm{~kg}$ $( \pm 6.7 \mathrm{~kg} ; 56.3 \pm 4.4$ control vs $50.8 \pm 7.5$ exercise). Eleven of 12 patients lost LBM over the 7-week course of CCRT, 4 from the control arm and 7 from the exercise arm. Losses in LBM during treatment ranged from $0.9 \mathrm{~kg}$ (exercise arm) to $11.3 \mathrm{~kg}$ (exercise arm). From treatment end until the 2-month posttreatment follow-up, 8 of 12 patients began to regain LBM (3 from the control arm and 5 from the exercise arm), resulting in a mean LBM of $54.5 \mathrm{~kg}( \pm 7.7 \mathrm{~kg} ; p=.01 ; 57.1 \pm 7.4$ control vs $52.3 \pm 7.9$ exercise) at the 2 -month posttreatment followup, a $2.4 \%$ increase from the end of CCRT.

\section{Changes in fat body mass}

Mean fat body mass (FBM) at baseline was $30.6 \mathrm{~kg}$ ( $\pm 7.5 \mathrm{~kg} ; 34.7 \pm 5.8$ control vs $27.6 \pm 7.5$ exercise), and, on average, accounted for $33 \%$ of TBM. On average, patients lost $11.1 \%(p=.001)$ of their FBM over the 7week course of therapy. Again, 11 of 12 patients lost FBM during the course of CCRT. The mean FBM at the end of treatment was $27.2 \mathrm{~kg}( \pm 6.4 \mathrm{~kg} ; 30.7 \pm 5.8$ control vs $24.7 \pm 5.9$ exercise). Unlike LBM, which began to return toward baseline after CCRT, FBM continued to decrease from the end of treatment until the 2-month posttreatment follow-up for 11 of 12 patients, 4 from the control arm and 7 from the exercise arm. By the 2-month follow-up appointment, on average, patients had lost $22.9 \%(p=.0003)$ of their baseline FBM. The loss in FBM from baseline to the 2-month follow-up appointment ranged from $2.3 \mathrm{~kg}$ (exercise arm) to $10.8 \mathrm{~kg}$ (control arm). The majority of FBM was lost from the android distribution. Overall, $7.8 \%(p=.004)$ of FBM was lost from the android distribution, whereas only $3.3 \%$ $(p=.01)$ was lost from the gynoid distribution.

\section{Changes in total body mass composition}

At baseline, mean total body composition was $64 \%$ LBM, 33\% FBM, and 3\% bone mass (Figure 2). Even though the weight loss continued over the 2 months after completion of CCRT, the total body composition ratio remained relatively unchanged over the course of treatment. At the end of treatment, the composition of TBM was nearly identical, with LBM accounting for $64 \%$ of TBM, FBM accounting for $32 \%$, and bone constituting $4 \%$. By the 2-month posttreatment follow-up, there was once again very little variation in total body composition, with TBM being 67\% LBM, 29\% FBM, and 4\% bone mass. A larger percentage of TBM lost over the course of CCRT was from LBM as compared to FBM (64\% vs $36 \%$, respectively). By the 1-month follow-up appointment, the contribution to TBM loss of LBM loss and FBM loss were quite similar (51\% vs $49 \%$, respectively). 


\section{A \\ Baseline}
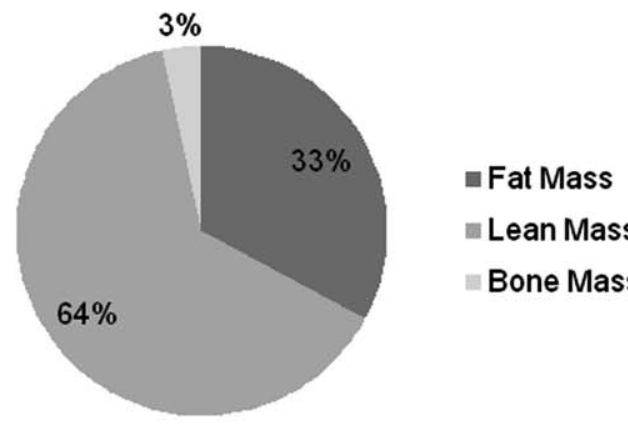

- Lean Mass

Bone Mass

\section{B End of CCRT}
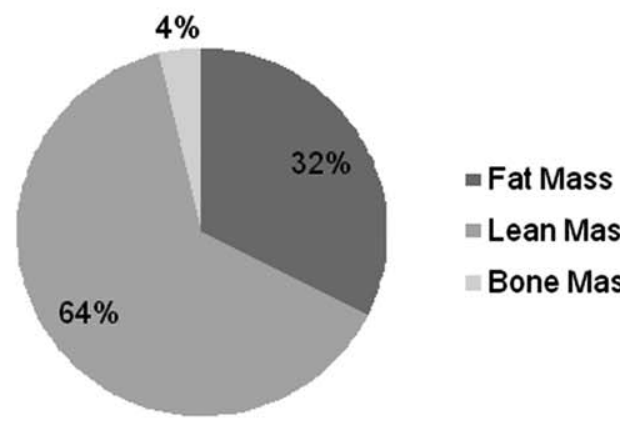

- Lean Mass

Bone Mass

\section{2-month follow -up}

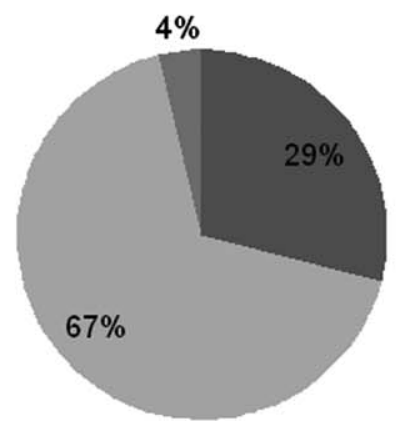

- Fat Mass

- Lean Mass

Bone Mass

FIGURE 2. (A) Percent of relative total body composition at baseline, (B) percent of relative total body composition at the end of concurrent chemoradiotherapy (CCRT), and (C) percent of relative total body composition at the 2-month posttreatment follow-up.

At the 2-month posttreatment follow-up, FBM loss contributed a larger percentage to TBM loss than LBM loss (60\% vs $40 \%$, respectively).

\section{Relationship of changes in lean and fat body mass to patient hydration status}

Through the course of the study, it was noted that patients who appeared to be clinically dehydrated because of treatment-related toxicity, also had changes noted on DEXA measurements of LBM. To better assess this relationship, we correlated serum creatinine obtained around the same time of DEXA scans to changes in LBM.

Mean baseline serum creatinine was $1.0 \mathrm{mg} / \mathrm{dL}$ (range, $0.7-1.2 \mathrm{mg} / \mathrm{dL}$ ). Mean serum creatinine increased to 1.5 $\mathrm{mg} / \mathrm{dL}$ (range, $0.7-3.8 \mathrm{mg} / \mathrm{dL} ; p=.07$ ) by the end of CCRT, and decreased to a mean value of $1.3 \mathrm{mg} / \mathrm{dL}$ (range, $0.8-2.3 \mathrm{mg} / \mathrm{dL} ; p=.2$ ) by the 2 -month posttreatment follow-up. DEXA measurements of LBM and serum creatinine were inversely associated $(r=-0.3 ; p=.04)$, however, such an association did not exist between FBM and serum creatinine $(r=-0.13 ; p=.4)$. Graphic representation of the relationship between changes in serum creatinine, LBM, and FBM can be found in Figure 3. The decrease in LBM seen over the course of treatment was inversely paralleled by an increase in serum creatinine. As LBM began to return toward baseline levels after treatment, serum creatinine began to normalize as well. This inverse relationship was not present for the changes in FBM, as FBM decreased at a nearly continuous rate from baseline to the 2-month posttreatment follow-up independently from changes in serum creatinine.

\section{DISCUSSION}

Using serial DEXA scans, we were able to characterize changes in total body composition during and after treatment for a group of patients receiving CCRT for locally advanced head and neck cancer. Nearly the entire cohort experienced significant weight loss from the initiation of CCRT to the end of treatment, with a mean weight loss over the 7-week course of treatment of $9.5 \mathrm{~kg}$. Of this weight loss, two-thirds was from a loss in LBM, and onethird was from a loss in FBM. Mean BMI declined from 29.6 to 26.5 during CCRT. After completion of CCRT, the majority of patients began to regain LBM; however, nearly all continued to lose FBM through the 2-month posttreatment follow-up period. Patients lost the majority of their FBM from the android distribution. BMI also continued to decline, with a mean BMI of 26.0 by the 2month posttreatment follow-up. The finding that FBM continued to decline post-CCRT may somewhat be accounted for by the fact that, on average, patients were overweight per BMI weight stratification at baseline. During CCRT, LBM loss exceeded FBM loss; however, the proportion of LBM and FBM loss compared to baseline LBM and FBM were similar. By treatment end, patients had lost $10.3 \%$ of their baseline LBM, and $11.1 \%$ of their baseline FBM. Thus, although LBM was the largest contributor to total mass lost at the end of treatment, this may be a reflection of the fact that LBM contributed more to TBM at baseline than FBM. This is supported by our finding that TBM composition was stable throughout our study, as the percentage contribution to TBM of LBM, FBM, and bone mass varied little from baseline to the 2-month posttreatment follow-up. LBM constituted 

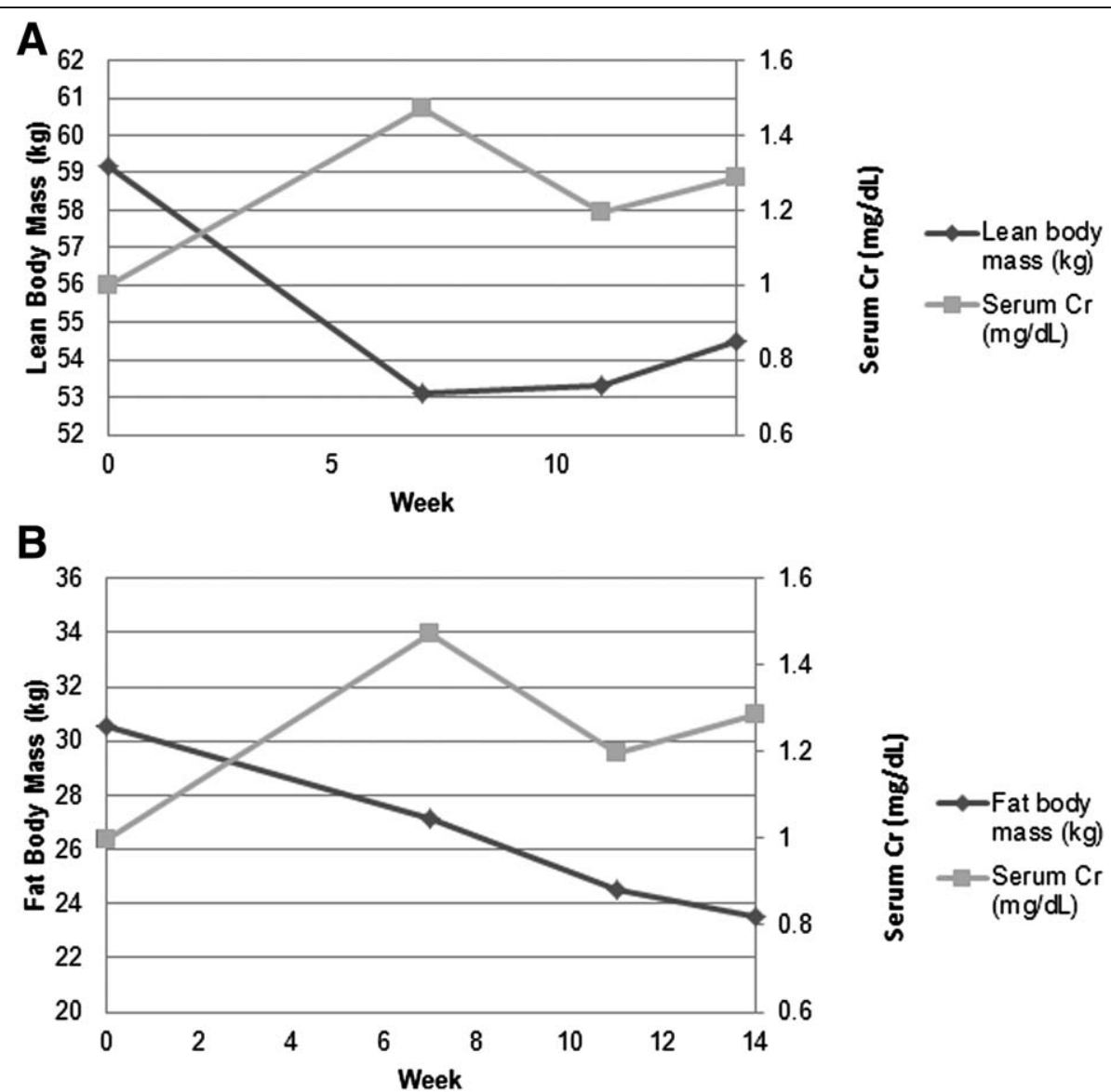

FIGURE 3. (A) Changes in mean lean body mass versus mean serum creatinine from baseline through the 2-month posttreatment follow-up. (B) Changes in mean fat body mass versus mean serum creatinine $(\mathrm{Cr})$ from baseline through the 2-month posttreatment follow-up.

$64 \%$ to $67 \%$ of TBM at baseline, at the end of CCRT, and at the 2-month posttreatment follow-up.

Our findings regarding changes in TBM and LBM for patients undergoing CCRT for locally advanced head and neck cancer are comparable to those reported by Silver et $\mathrm{al}^{6}{ }^{6}$ who reported a $10.1 \mathrm{~kg}$ loss in TBM at 1 -month posttreatment, with $71.7 \%$ of this loss accounted for by loss in LBM. We found a slightly greater loss in TBM with less contribution of LBM in the present analysis $(12.0 \mathrm{~kg}$ loss in TBM, 49\% LBM). A second difference between our findings and those reported by Silver et $\mathrm{al}^{6}$ is that patients in our cohort began to regain LBM after completion of CCRT, and this was evident at both the 1-month and 2-month posttreatment follow-ups, whereas Silver et $\mathrm{al}^{6}$ reported a continuous linear decline in LBM at the 1month posttreatment follow-up. Silver et $\mathrm{al}^{6}$ only reported a 1-month posttreatment follow-up, and, thus, we cannot compare our findings at 2-months posttreatment followup. It is possible that differences in our findings have underestimated the degree of LBM loss because of 7 patients receiving exercise training during and after CCRT; however, there were no statistically significant differences in changes of total body composition between this group and the control group, although this is not surprising given the overall small sample size.

Another finding in our study was the inverse correlation between LBM loss and serum creatinine during and after
CCRT. Several plausible explanations exist for this correlation. First, patients undergoing CCRT for head and neck cancer often become significantly dehydrated. ${ }^{19}$ This volume depletion can lead to prerenal azotemia, which can result in increased serum creatinine. ${ }^{20}$ Furthermore, water constitutes a large percentage of LBM. Mean LBM reached its nadir at the end of treatment, which corresponded with the greatest mean increase in serum creatinine. After treatment, when one would expect volume status to improve, LBM began to rebound and serum creatinine levels began to normalize. Thus, a portion of the inverse correlation between serum creatinine and LBM may merely be a reflection of hydration status. Another explanation for the rise in serum creatinine during treatment is secondary to renal toxicity from the platinumbased chemotherapy regimen. ${ }^{21}$ This could explain the acute rise in creatinine during treatment and why serum creatinine began to normalize post-CCRT, but mean baseline creatinines were never reached.

The possibility that at least a portion of LBM loss observed on DEXA may merely be a reflection of fluid volume status raises the question as to whether DEXA is the optimal tool to measure body composition in patients with cancer with fluctuating fluid status, and who during treatment can be dehydrated or hypovolemic. ${ }^{22}$ Going et $\mathrm{al}^{23}$ assessed the ability of DEXA to detect changes in body composition in 17 patients undergoing a 
dehydration-rehydration protocol that induced small changes in body composition. They concluded that although DEXA seemed to provide accurate estimates of changes in body water during the dehydration-rehydration protocol that it was limited in determining whether these changes occurred in the lean or fat components of TBM. Thus, although these results show potential for DEXA in detecting small changes in hydration status, less is known regarding the overall accuracy of DEXA in patients undergoing large changes in volume status, such as in patients with head and neck cancer undergoing treatment. More recently, CT has been used as a means of accurately assessing $\mathrm{LBM}$ in patients with cancer. ${ }^{24,25} \mathrm{CT}$ offers a benefit of convenience for the patient and limits additional tests as most patients with cancer undergo routine imaging with either CT or MRI before and after treatment. Future work is needed to define the ideal modality for measuring changes in LBM for patients with cancer undergoing therapy that may result in significant changes in overall hydration status. A direct comparison between CT and DEXA in such a patient group may help to shed more light on this issue.

Seventy-five percent of the patients in this study received a PEG tube before or during treatment $(50 \%$ before and $25 \%$ during) for nutritional support, and all patients received nutritional guidance from a dietician before the initiation of treatment. The proportion of our patients receiving a PEG tube before or during treatment are greater than those recently reported by Locher et $\mathrm{al}^{26}$ through analysis of the Surveillance, Epidemiology, and End Results database, in which $47.8 \%$ of patients treated for head and neck cancer with radiotherapy received a PEG tube before or during treatment $(30 \%$ before and $70 \%$ during). The higher use of PEG tube placement in our study is consistent with findings by Murphy et $\mathrm{al}^{27}$ that showed that PEG tube placement occurs more commonly in academic centers as compared to community settings. Despite the majority of patients receiving nutritional supplementation with a PEG tube, our study shows that patients with head and neck cancer receiving CCRT experience significant TBM, LBM, and FBM losses. Importantly, weight loss during treatment of advanced head and neck cancer has been shown to be independently associated with decreased overall survival. ${ }^{18}$ Therefore, identifying means by which to diminish this weight loss is of utmost clinical importance. A combination of extended nutritional counseling and or supplementation with combined exercise training may help mitigate the loss of LBM and fat during and after CCRT. ${ }^{28}$ Exercise in cancer survivors has been shown to improve functionality and QOL in patients undergoing treatment for cancer. $^{29,30}$ A recent Cochrane review found that exercise training during cancer treatment may improve overall health-related QOL, and that increasing intensities of exercise were related to improved benefit. ${ }^{30}$ Furthermore, in patients with breast and prostate cancers, exercise training has been shown to increase or preserve LBM. $^{31,32}$ Nutritional interventions are also recommended for patients with head and neck cancer. A recent systematic review supported the use of individualized dietary counseling in combination with dietary supplementation for patients with head and neck cancer treated with radiotherapy. ${ }^{33}$ Therefore, a combined exercise and nutritional program may be able to provide the same benefits for patients undergoing CCRT for locally advanced head and neck cancer. The challenge becomes identifying the ideal balance between nutritional support and exercise, as well as the timing of these interventions, that provides optimal patient outcomes. A recent review of Radiation Therapy Oncology Group 90-03 identified an association between nutritional support before initiation of treatment and worse outcomes after treatment, even when controlling for stage and performance status. ${ }^{34}$ Although these findings are controversial and require validation, they do highlight that further work is needed to identify the preferred timing and method of nutritional support for patients receiving treatment for head and neck cancer. Similar efforts are needed to identify the ideal timing and mode of exercise training, and to better define the interplay between exercise training and nutritional support.

In conclusion, we show that patients with locally advanced squamous cell head and neck cancer lose a significant amount of TBM, LBM, and FBM while undergoing CCRT. LBM begins to return toward baseline after the completion of treatment for most patients, whereas FBM loss continued through the 2-month posttreatment follow-up. Changes in LBM as measured by DEXA were inversely related to changes in serum creatinine suggesting that in patients prone to dehydration, DEXA scan measurements of total body composition may lead to inaccuracies. However, the loss of LBM in patients with head and neck cancer undergoing CCRT is apparent and its implications regarding patient's functional status and QOL need to be studied further. Exercise training during and after cancer treatment has been effective in negating LBM loss in other patients with cancer, and may offer an opportunity to do the same for patients with head and neck cancer, thereby helping to improve patient QOL and outcomes.

\section{Acknowledgments}

We thank Debra Arnoldi for her work as the clinical research coordinator and her contribution to data collection. We additionally thank Joanna Jennens, Eric Pear, Ayowal Oladeji, Brad Grincewicz and Michelle Baumgart for their work in conducting physical training and mobility assessments.

\section{REFERENCES}

1. Awad S, Tan BH, Cui H, et al. Marked changes in body composition following neoadjuvant chemotherapy for oesophagogastric cancer. Clin Nutr 2012;31:74-77.

2. Fouladiun M, Körner U, Bosaeus I, Daneryd P, Hyltander A, Lundholm KG. Body composition and time course changes in regional distribution of fat and lean tissue in unselected cancer patients on palliative care-correlations with food intake, metabolism, exercise capacity, and hormones. Cancer 2005; 103:2189-2198.

3. Gil KM, Frasure HE, Hopkins MP, Jenison EL, von Gruenigen VE. Body weight and composition changes in ovarian cancer patients during adjuvant chemotherapy. Gynecol Oncol 2006;103:247-252.

4. Harvie MN, Campbell IT, Thatcher N, Baildam A. Changes in body composition in men and women with advanced nonsmall cell lung cancer (NSCLC) undergoing chemotherapy. J Hum Nutr Diet 2003;16:323-326.

5. Jacquelin-Ravel N, Pichard C. Clinical nutrition, body composition and oncology: a critical literature review of the synergies. Crit Rev Oncol Hematol 2012;84:37-46.

6. Silver HJ, Dietrich MS, Murphy BA. Changes in body mass, energy balance, physical function, and inflammatory state in patients with locally advanced head and neck cancer treated with concurrent chemoradiation after low-dose induction chemotherapy. Head Neck 2007;29:893-900. 
7. Tan BH, Birdsell LA, Martin L, Baracos VE, Fearon KC. Sarcopenia in an overweight or obese patient is an adverse prognostic factor in pancreatic cancer. Clin Cancer Res 2009;15:6973-6979.

8. Smith MR. Changes in fat and lean body mass during androgen-deprivation therapy for prostate cancer. Urology 2004:63:742-745.

9. Demark-Wahnefried W, Peterson BL, Winer EP, et al. Changes in weight, body composition, and factors influencing energy balance among premenopausal breast cancer patients receiving adjuvant chemotherapy. J Clin Oncol 2001;19:2381-2389.

10. Fearon K, Strasser F, Anker SD, et al. Definition and classification of cancer cachexia: an international consensus. Lancet Oncol 2011;12:489-495.

11. Wallengren O, Lundholm K, Bosaeus I. Diagnostic criteria of cancer cachexia: relation to quality of life, exercise capacity and survival in unselected palliative care patients. Support Care Cancer 2013;21:1569-1577.

12. Fearon KC, Voss AC, Hustead DS; Cancer Cachexia Study Group. Definition of cancer cachexia: effect of weight loss, reduced food intake, and systemic inflammation on functional status and prognosis. Am J Clin Nutr 2006;83:1345-1350.

13. Couch M, Lai V, Cannon T, et al. Cancer cachexia syndrome in head and neck cancer patients: part I. Diagnosis, impact on quality of life and survival, and treatment. Head Neck 2007;29:401-411.

14. Tsai S. Importance of lean body mass in the oncologic patient. Nutr Clin Pract 2012;27:593-598.

15. Chasen MR, Bhargava R. A descriptive review of the factors contributing to nutritional compromise in patients with head and neck cancer. Support Care Cancer 2009;17:1345-1351.

16. Jager-Wittenaar H, Dijkstra PU, Vissink A, et al. Changes in nutritional status and dietary intake during and after head and neck cancer treatment. Head Neck 2011;33:863-870.

17. Adelstein DJ, Saxton JP, Lavertu P, et al. A phase III randomized trial comparing concurrent chemotherapy and radiotherapy with radiotherapy alone in resectable stage III and IV squamous cell head and neck cancer: preliminary results. Head Neck 1997;19:567-575.

18. Mick R, Vokes EE, Weichselbaum RR, Panje WR. Prognostic factors in advanced head and neck cancer patients undergoing multimodality therapy. Otolaryngol Head Neck Surg 1991;105:62-73.

19. Ang KK, Harris J, Garden AS, et al. Concomitant boost radiation plus concurrent cisplatin for advanced head and neck carcinomas: radiation therapy oncology group phase II trial 99-14. J Clin Oncol 2005;23:3008-3015.

20. Rahman M, Shad F, Smith MC. Acute kidney injury: a guide to diagnosis and management. Am Fam Physician 2012;86:631-639.

21. Madias NE, Harrington JT. Platinum nephrotoxicity. Am J Med 1978;65: 307-314.
22. Berk L, Rana S. Hypovolemia and dehydration in the oncology patient. $J$ Support Oncol 2006;4:447-454; discussion 455-457.

23. Going SB, Massett MP, Hall MC, et al. Detection of small changes in body composition by dual-energy x-ray absorptiometry. Am J Clin Nutr 1993;57: 845-850.

24. Mourtzakis M, Prado CM, Lieffers JR, Reiman T, McCargar LJ, Baracos VE. A practical and precise approach to quantification of body composition in cancer patients using computed tomography images acquired during routine care. Appl Physiol Nutr Metab 2008;33:997-1006.

25. Parsons HA, Baracos VE, Dhillon N, Hong DS, Kurzrock R. Body composition, symptoms, and survival in advanced cancer patients referred to a phase I service. PLoS One 2012; 7:e29330.

26. Locher JL, Bonner JA, Carroll WR, et al. Gastrostomy tube placement and use in patients with head and neck cancer. Head Neck 2012;34:422428.

27. Murphy BA, Chen A, Curran WJ Jr, et al. Longitudinal oncology registry of head and neck carcinoma (LORHAN): initial supportive care findings. Support Care Cancer 2009;17:1393-1401.

28. Al-Majid S, Waters H. The biological mechanisms of cancer-related skeletal muscle wasting: the role of progressive resistance exercise. Biol Res Nurs 2008;10:7-20.

29. Segal RJ, Reid RD, Courneya KS, et al. Resistance exercise in men receiving androgen deprivation therapy for prostate cancer. J Clin Oncol 2003; $21: 1653-1659$.

30. Mishra SI, Scherer RW, Snyder C, Geigle PM, Berlanstein DR, Topaloglu $\mathrm{O}$. Exercise interventions on health-related quality of life for people with cancer during active treatment. Cochrane Database Syst Rev 2012;8: CD008465.

31. Courneya KS, Segal RJ, Mackey JR, et al. Effects of aerobic and resistance exercise in breast cancer patients receiving adjuvant chemotherapy: a multicenter randomized controlled trial. J Clin Oncol 2007;25:43964404.

32. Galvão DA, Nosaka K, Taaffe DR, et al. Resistance training and reduction of treatment side effects in prostate cancer patients. Med Sci Sports Exerc 2006;38:2045-2052.

33. Garg S, Yoo J, Winquist E. Nutritional support for head and neck cancer patients receiving radiotherapy: a systematic review. Support Care Cancer 2010;18:667-677.

34. Rabinovitch R, Grant B, Berkey BA, et al. Impact of nutrition support on treatment outcome in patients with locally advanced head and neck squamous cell cancer treated with definitive radiotherapy: a secondary analysis of RTOG trial 90-03. Head Neck 2006;28:287296 